\section{Cahiers de Narratologie}

Analyse et théorie narratives

15 | 2008

Récits et genres historiques

\title{
Le regard anachronique
}

(Goya vu par Saura)

Jean-Paul Aubert

\section{OpenEdition}

Journals

\section{Édition électronique}

URL : http://journals.openedition.org/narratologie/716

DOI : $10.4000 /$ narratologie. 716

ISSN : 1765-307X

Éditeur

LIRCES

\section{Référence électronique}

Jean-Paul Aubert, «Le regard anachronique », Cahiers de Narratologie [En ligne], 15 | 2008, mis en ligne le 10 janvier 2011, consulté le 06 mai 2019. URL : http://journals.openedition.org/narratologie/716 ; DOI : 10.4000/narratologie.716

Ce document a été généré automatiquement le 6 mai 2019.

\section{(c) $($ i) $(9)$}

Cahiers de Narratologie - Analyse et théorie narratives est mis à disposition selon les termes de la licence Creative Commons Attribution - Pas d'Utilisation Commerciale - Pas de Modification 4.0 International. 


\title{
Le regard anachronique
}

(Goya vu par Saura)

\author{
Jean-Paul Aubert
}

1 Suggérer - comme nous le faisons dans le titre de cette communication - qu'une biographie historique (genre auquel on est tenté a priori de rattacher le film Goya en Burdeos ${ }^{1}$ dont il sera question dans ces lignes) puisse tirer sa valeur de ses anachronismes peut surprendre. Le genre du film historique - dont les codes ont été durablement fixés par la tradition hollywoodienne - tient en effet l'anachronisme pour une faute impardonnable. Toutefois, les déclarations de Carlos Saura, qui s'est défendu à plusieurs reprises d'avoir voulu réaliser un film naturaliste et qui est même allé jusqu'à récuser l'appartenance de Goya en Burdeos au genre de la biographie historique, nous invitent à nous intéresser à cette œuvre à partir des licences que son auteur prend avec l'Histoire. Non point évidemment pour nous en offusquer, mais afin d'essayer de comprendre en quoi l'anachronisme, parfaitement assumé, sert le projet esthétique de Carlos Saura dans Goya en Burdeos et apparaît en définitive comme une manière originale et ambitieuse de penser l'Histoire de l'art.

\section{Anachronismes}

2 Avant d'aborder plus précisément le film Goya en Burdeos, que l'on nous permette d'envisager d'un point de vue plus général cette question certainement essentielle de la relation complexe que le cinéma de reconstitution historique entretient avec l'Histoire.

Le Dictionnaire historique de la langue française donne du terme "anachronisme" la définition suivante :

Anachronisme : « est formé en français (1625) à partir des éléments grecs ana « en arrière » et khronos « temps ». Le mot désigne donc les confusions entre un fait et une époque plus ancienne [...], anachronisme s'applique à toute confusion chronologique et à tout décalage dans le temp. Le dérivé anachronique a des valeurs correspondantes. Plus usuel que le substantif, il signifie surtout « qui correspond à un écart, à une erreur de temps et d'époque $»^{2}$. 
Le Dictionnaire culturel en langue française définit l'anachronisme comme une "confusion de dates, [l'] attribution à une époque de ce qui appartient à une autre» et cite Bossuet pour qui les anachronismes sont « cette erreur qui fait confondre les temps $»^{3}$. La notice s'achève sur l'exemple de quelques lieux où fleurissent les anachronismes : tableau ou roman historique. A la lecture des différentes notices que lui consacrent les dictionnaires, on comprend que l'anachronisme se décline généralement selon deux modalités: l'erreur de date et le jugement porté sur le passé à partir de l'époque contemporaine. "Confusion ", « décalage ", " écart ", « erreur » : l'anachronisme a donc mauvaise réputation. Toutefois, la sémantique est parfois facétieuse au point de nous faire oublier que l'anachronisme est constitutif de la relation à un objet passé. L'historien même, dont tous les efforts sont tendus afin d'éviter ce qui constitue une faute professionnelle, ne peut s'y soustraire : c'est depuis le temps présent qu'il contemple le passé. Michel de Certeau a montré combien l'écriture historiographique est toujours révélatrice de son temps aussi bien par le choix de son objet que par les modalités scripturaires qu'elle emprunte pour le décrire ${ }^{4}$. C'est une véritable loi qui s'impose à l'historien et qui s'explique selon Paul Ricœur par le fait que l'accès à la connaissance du passé n'est rendu possible que par la présence du passé dans le présent :

C'est d'abord comme héritiers que les historiens se placent à l'égard du passé avant de se poser en maîtres artisans des récits qu'ils font du passé. Cette notion d'héritage présuppose que d'une certaine façon le passé se perpétue dans le présent et ainsi l'affecte ${ }^{5}$.

5 La réflexion de Ricœur ne débouche cependant pas sur une posture relativiste qui ferait douter du statut de connaissance de l'Histoire et ne verrait dans le discours historique qu'une catégorie du discours fictionnel. Car, pour Ricœur, si les récits d'histoire partagent avec les récits de fiction plus d'un trait commun, ils s'en distinguent au moins sur un point essentiel qui est celui de l'«intentionnalité de connaissance ». L'« opération historiographique » (selon l'expression de Michel de Certeau) vise en effet à produire un énoncé scientifique fondé sur le traitement rigoureux des sources, sur la construction d'hypothèses et sur l'élaboration d'un savoir vérifiable par tous. La création artistique a théoriquement tout loisir de s'affranchir des contraintes de la production scientifique. Si les limites de la documentation brident l'ambition de l'historien, elles devraient stimuler au contraire celle du poète qui n'est pas tenu par l'intentionnalité de connaissance et qui reste libre quant à lui d'inventer ce qu'il ignore. C'est une liberté dont le cinéma se saisit peu ou, du moins, qu'il ne revendique que rarement. Bien au contraire, l'obsession de l'authenticité, imposée par les studios hollywoodiens à l'âge classique, s'est largement perpétuée jusqu'à notre époque et n'a été remise en cause que de manière marginale. Hollywood a prôné le respect de la "vérité historique ", n’hésitant pas, ainsi que le remarque Michel Cieutat, à « transformer les cinémas en salles de classe » à l'occasion de spectacles qui "combinaient l'art d'enseigner et celui de distraire $»^{6}$. Cette supposée capacité du cinéma d'écrire l'histoire avec la rigueur de l'historien est même devenue argument publicitaire. Dans les dossiers de presse, les réalisateurs revendiquent la caution de scientifiques de renom, citent les livres savants dont ils se sont inspirés, énumèrent les noms des conseillers prestigieux qui les ont guidés. Si bien que ce n'est pas l'inventivité du cinéaste qu'une partie de la critique cinématographique a pris l'habitude d'évaluer, mais au contraire sa capacité à restituer « la vérité » d'une époque.

6 Le récent film que Mel Gibson consacre à la civilisation Maya, Apocalypto (2007), et la polémique qu'il a engendrée sont à cet égard exemplaires. Dans les entretiens qu'il 
accorde à la presse, le réalisateur américain met l'accent sur le travail de documentation acharné censé garantir l'authenticité des costumes, des objets, des tatouages et des modes de vie des indigènes. Mel Gibson, qui avait fait parler en araméen les protagonistes de sa Passion du Christ, a même sollicité des linguistes afin qu'ils traduisent les dialogues d' Apocalypto dans un idiome se rapprochant du yucatèque tel qu'il était parlé à l'époque où est situé le film, le XVe siècle. Poudre aux yeux que tout cela rétorquent certains scientifiques, qui récusent ce mélange artificiel de yucatèque ancien et contemporain. D'autres s'indignent de voir l'Histoire mise à mal dans un film qui confond Mayas et Aztèques, situe la décadence de l'empire maya au moment de la colonisation espagnole et fut tourné dans des paysages qui n'ont rien de semblable avec ceux du Yucatan. D'autres encore s'étonnent de ce que les quinze mille figurants recrutés pour l'occasion n'appartiennent pas à l'ethnie maya.

La reconstitution naturaliste, en revendiquant une «intentionnalité de connaissance », en affichant sa prétention illusoire à offrir le spectacle « réaliste » d'un événement ou d'un fait historique, en dissimulant en somme son statut de représentation, s'expose naturellement à la critique du scientifique. Les foudres que s'est attirées le film Apocalypto ne sont finalement que le reflet d'une polémique aussi stérile qu'éternelle qui met en concurrence l'inventivité du cinéaste et la rigueur de l'historien. Tant les revendications d'authenticité du cinéaste que les cris outragés des historiens entretiennent une confusion sur la véritable nature de ce type de film. Mieux vaudrait reconnaître d'emblée que la reconstitution historique sert dans ce cas, comme dans beaucoup d'autres, d'alibi culturel à ce qui n'est au fond qu'un spectacle destiné à satisfaire la soif d'exotisme du spectateur.

8 Paradoxalement, ces procès en anachronisme où l'on reproche aux films de ne pas tenir leurs promesses démontrent la difficulté pour le cinéma en costume de s'émanciper des contraintes du naturalisme. Ceux qui, au nom de la vérité historique, s'en font les procureurs feignent d'ignorer que le cinéma ne peut offrir du passé qu'une représentation. La spécificité iconique du médium cinématographique est de ce point de vue décisive. Tandis que le texte écrit nous livre avec des mots une interprétation du passé, le cinéma nous la donne à voir. Il ne reproduit pas des faits historiques, il les transpose en image, il les met en scène, il les recrée selon ses codes et avec les moyens qui lui sont propres, en un mot il les représente. On comprend dès lors que le cinéma réaliste, faute de pouvoir prétendre offrir la vérité historique, s'attache, sans oser l'avouer, à créer les apparences de cette vérité, ce que l'on désigne le plus souvent du terme de vraisemblance. Or ce qui peut sembler un anachronisme aux yeux de l'historien peut apparaitre comme parfaitement acceptable du point de vue d'un spectateur moins averti, et inversement. Par ailleurs l'avancée de l'historiographie rendra invraisemblable demain ce qui semble vraisemblable aujourd'hui. Mais qu'importe, en tant que produit industriel de grande consommation, un film historique se voit dans l'obligation d'offrir, au moment de sa sortie et au public le plus large possible une image du passé qui ne s'éloigne pas de ses schémas de représentation. Attribuer au discours fictionnel l'intentionnalité de connaissance du discours historique, c'est donc confondre l'objet du discours historique qui est le passé et l'objet du discours fictionnel qui est en fait, dans l'immense majorité des films, le passé tel que le public se l'imagine. Ce qui revient à dire que l'anachronisme est inhérent au film historique. Si Goya en Burdeos présente quelque originalité à nos yeux, ce n'est donc pas parce que le regard qu'il porte sur le temps de Goya est anachronique -il ne peut en être autrement - mais parce que ce regard anachronique, pleinement assumé, 
participe d'une conception originale du temps dont nous allons essayer de dessiner les grandes lignes

\section{Petits arrangements avec l'Histoire}

9 《Le concept général est de faire un film sur Goya éloigné du naturalisme » ${ }^{7}$ écrit Saura en guise d'introduction au scénario de $1996^{\circ}$. Ailleurs il affirme : "Les libertés que je me suis autorisées dans mon Goya sont la conséquence de ma vision personnelle du personnage. Je n'ai pas voulu faire un film historique, ni le résumé de son œuvre, encore moins une analyse psychologique du personnage - même si tout cela se trouve dans mon film, mais j'ai voulu extraire d'une longue existence les images qui me semblaient essentielles $»^{9}$. Dans cette dernière citation, la revendication de la liberté de création est manifeste: «ma vision personnelle», «je n'ai pas voulu», «les libertés que je me suis autorisées ». Une façon d'affirmer que Goya en Burdeos ne répond pas à une « intentionnalité de connaissance », mais s'inscrit dans un projet artistique personnel et original, étranger à toute démarche scientifique. Goya en Burdeos est bel et bien le Goya de Saura. Ce n'est pas un hasard si celui-ci utilise l'expression « mon Goya» dans un excès un brin vaniteux de possessivité peut-être, mais qui est davantage l'affirmation d'une proximité, d'une intimité même entre le cinéaste et le peintre.

Les déclarations d'intention de Carlos Saura invitent le spectateur à mesurer la créativité du cinéaste à l'aune des libertés qu'il s'autorise avec l'Histoire. Les nombreuses études dont le film a déjà fait l'objet n'ont pas manqué de relever un certain nombre d'écarts à l'égard de ce que les spécialistes du dix-neuvième siècle espagnol tiennent pour des faits avérés. Nous ne referons pas ici l'inventaire laborieux de ces menus accommodements avec l'Histoire. Admettons que la présence d'un Godoy déjà mûr lors d'une réception chez les ducs d'Osuna, située à une époque où le futur favori n'est encore qu'un enfant, ou le charmant accent italien dont Bayeu se voit affublé peuvent laisser perplexe. Maladresse dans le premier cas? effet secondaire de la coproduction italienne dans le second? Rien n'est certain. En revanche, le choix de faire de Rosarito la fille de Goya alors que la plupart des historiens s'accordent à dire aujourd'hui que cette relation filiale n'est pas avérée ne saurait être mis sur le compte d'une quelconque négligence. La présence de la jeune Rosarito aux côtés du vieux peintre relève de la stratégie narrative qui consiste à accompagner la figure dont on prétend brosser le portrait d'un interlocuteur auquel il livre ses souvenirs ou d'un confident auprès de qui il s'épanche. Une facilité, sans doute, mais qui permet d'éviter le recours systématique à l'artifice et aux lourdeurs du soliloque ou de la voix-off. On pourra objecter le fait qu'un autre personnage, Antonio de Brugada en l'occurrence, aurait pu tenir lieu d'interlocuteur crédible de Goya. La présence du jeune peintre aux côtés du vieux maître est attestée; Saura ne l'ignore pas. Mais on imagine aisément le parti qu'il pouvait tirer de la mise en scène du couple naturellement attachant du vieillard et de sa jeune enfant. Le cinéaste donnait à ce duo une dimension affective que n'aurait pas eu sans doute celui formé par Goya et Antonio de Brugada. Par ailleurs, la relation de filiation ainsi établie permettait de renforcer un thème qui est au cœur du film, celui de la transmission ou de "l'art d'hériter » comme le dit joliment Nancy Berthier ${ }^{10}$. Faute de s'appuyer sur une documentation historique fiable, la proximité et la connivence entre les deux personnages gagnent finalement en vraisemblance grâce au choix des interprètes, Francisco Rabal et Dafné Fernándes, qui reconstituent pour Goya en Burdeos le couple qu'ils avaient déjà formé dans un film 
précédent de Carlos Saura, Pajarico (1997). La relation entre les deux personnages apparaitt comme d'autant plus crédible aux yeux du spectateur qu'elle a déjà été mise en scène en des termes comparables dans un autre film. La réalité des faits se voit ainsi sacrifiée sur l'autel de l'effet de réel. Saura se contente, pour sa part, de justifier cette entorse à la vérité historique en expliquant que Goya considérait effectivement Rosarito comme sa fille.

11 La relation amoureuse entre Goya et Cayetana constitue l'autre élément structurant du récit. On sait que les historiens ont des doutes sur la réalité de la relation intime entre le peintre et la duchesse. Mais la légende de la passion amoureuse interrompue par l'empoisonnement de Cayetana, forgée par la tradition romantique, s'est perpétuée jusqu'à nos jours. Saura y souscrit et, au risque de déplaire à l'historien sourcilleux, comble les attentes d'un large public qui tient ce qui n'est peut-être qu'une légende pour la stricte vérité historique. Interrogé sur cette interprétation, Saura revendique sa liberté d'imaginer : "Qu'ils aient eu ou non une liaison? Qu'est-ce que cela peut faire ? [...] ce film est un produit de l'imagination [...] $»^{11}$.

12 Autour du couple du vieillard et de l'enfant gravite une galaxie de personnages que l'on est tenté de qualifier de secondaires. Le système des personnages élaboré par Saura tend généralement à estomper la personnalité des figures qui composent l'entourage de Goya. Cet effacement est particulièrement remarquable s'agissant du personnage de Leocadia. De celle qui fut la dernière compagne de Goya, les témoins de l'époque (Moratín en particulier) rapportent qu'elle était dotée d'un caractère passionné et intransigeant. Saura réduit Leocadia Weiss à une présence affectueuse et discrète. D'une manière générale, les portraits que le cinéaste brosse des proches de Goya tiennent de l'esquisse voire dans certains cas de la caricature: Moratin est un bourgeois aimable, Bayeu un mauvais peintre que guette le ridicule, Godoy un arriviste infatué. De même le monde courtisan que Goya fréquenta avec assiduité se limite à quelques figures rapidement esquissées, ce qui a pour effet de minorer l'importance des relations que l'artiste avait entretenues avec les hauts personnages de son temps. Un tel parti pris qui a pour effet d'édulcorer le parcours courtisan de Goya semble inhérent au genre de la biographie qui tend à braquer les projecteurs sur une figure jugée exceptionnelle au détriment de ses contemporains ramenés au rang de comparses ou de faire-valoir.

13 Ces licences prises avec l'histoire ne sont donc pas imputables à un défaut de documentation. Du reste, Saura dit avoir lu tout ce qui s'est écrit sur Goya et cite plus particulièrement l'ouvrage de Jacques Fauqué et Ramón Villanueva Etcheverria dont il reconnaît qu'il fut sa principale source de connaissance ${ }^{12}$. C'est pourquoi les écarts à l'égard de ce que les historiens nous révèlent du parcours artistique et vital du peintre relèvent davantage pour les cas que nous venons d'envisager d'une volonté de souscrire à la vision romantique de Goya et de resserrer le récit autour de ce personnage selon une stratégie narrative partagée par nombre de biographies historiques. Hormis peut-être aux yeux des spécialistes de l'époque, les libertés prises avec l'histoire, en ce qui concerne en particulier les rôles attribués à Rosarito et à Cayetana, renforcent plus qu'elles n'altèrent la vraisemblance des personnages et de l'action.

\section{Anachronie}

Ces petits arrangements avec l'histoire ne permettent pas à Goya en Burdeos de se distinguer fondamentalement des biographies filmées habituelles. On pourrait dire qu'ils 
relèvent de cet anachronisme constitutif de la reconstitution historique. L'originalité de Goya en Burdeos vient d'ailleurs et en particulier de son élaboration du temps. Une analyse des relations entre le temps de l'histoire et le temps du récit place clairement le film dans le registre de l'anachronie. Le projet initial consistait à élaborer le récit selon le principe de la machine à remonter le temps en commençant par la mort de Goya puis en remontant jusqu'à sa naissance. L'idée fut en partie abandonnée. On imagine que Saura fut rebuté par la trop grande rigidité d'une formule qui revenait à inverser la flèche du temps sans que cela n'entame la logique historique d'un avant et d'un après alors que la liberté que Saura voulait prendre avec l'Histoire lui dictait de s'émanciper des rigueurs de la chronologie. D'où une perturbation du continuum temporel qui contredit la logique des événements et entrave parfois la compréhension de ces derniers. C'est ainsi que les séquences 1, 2, 3 et 4 nous situent en 1827, la séquence 5 en 1781, la séquence 6 en 1828, les séquences 7,8 et 9 en 1792-1793, la séquence 10 en 1827-1828, les séquences 11,12, 13 et 14 en 1798, etc. On est là très loin de la biographie classique qui, selon les termes de Yannick Mouren, se limite à « raconter la vie du personnage célèbre de manière linéaire d'un point $\mathrm{A}$ de sa vie à un point $\mathrm{Z}$ de sa mort $\aleph^{13}$. Saura a fui cette linéarité qui permet de faire avancer le récit selon la stricte logique de la succession qui veut que chaque nouvelle séquence soit la conséquence de la précédente et justifie la suivante. Il s'est écarté résolument de l'ordre chronologique qui selon le principe du post hoc, ergo propter hoc, cher à la biographie classique, semble substituer au hasard la nécessité et associe implicitement à l'existence d'un grand homme l'idée d'un destin à accomplir. A une narration linéaire, il a préféré cette narration au tracé incertain, faite d'allers et retours permanents entre passé et présent, et dont le principe est explicité par l'image de la spirale que le peintre dessine à trois reprises et dont il dit que «son tracé est aventureux » («su trazado es aventurero »).

15 A l'absence de succession s'ajoute une indifférenciation des époques. La séquence 27, censée se dérouler dans les jardins d'Aranjuez, à une époque où le jeune peintre réalisait des cartons à partir desquels la Manufacture Royale de Tapis confectionnait les tapisseries destinées à orner le palais du Pardo, mêle plusieurs temps puisqu'elle réunit en un seul lieu et en un même instant les femmes que Goya connut à des époques différentes. La confusion est renforcée du fait que la séquence s'organise autour de la découverte macabre de Rosario noyée dans un bassin du parc alors que la jeune femme survivra plusieurs années à Goya. Au lieu de se succéder, les différentes régions du passé coexistent. Des personnages qui, dans la réalité, ne se sont peut-être jamais croisés, entrent sur la scène de ce théâtre mental qu'est l'imagination de Goya. Les différentes régions du passé coexistent encore d'une manière encore plus explicite dans les séquences où se côtoient Goya âgé interprété par Francisco Rabal et Goya jeune joué par José Coronado. Ou dans ce plan sur la main du peintre qui ébauche sur un carnet le croquis d'une silhouette dénudée, une main dont le spectateur ne peut décider si elle appartient au jeune homme qui jadis croquait la duchesse ou au vieillard désormais égaré dans ses souvenirs. La passion amoureuse semble avoir aboli le temps. Cette abolition du temps va déboucher dans la dernière séquence sur une sorte de synthèse du cycle vital où se confondent les différents âges du personnage y compris sa naissance et sa mort.

Le travail sur les décors et la lumière sert l'entreprise de Saura. Sculpté par la lumière de Vittorio Storaro, l'espace semble à géométrie variable, judicieusement dessiné en outre à partir de cloisons tantôt opaques, tantôt transparentes. Ce travail sur la lumière et sur le décor permet une circulation extrêmement libre des personnages qui se meuvent avec 
légèreté, se glissant sans obstacle d'une pièce à l'autre au mépris de tout réalisme. On peut y voir la traduction spatiale de la fluidité du temps qui permet au film de passer d'une époque à une autre sans que l'on ait la sensation d'une rupture. Sans changement de plan, sans mouvement de caméra, espaces et époques communiquent comme dans cette séquence où après avoir découvert Les Ménines de Velázquez, Goya jeune traverse un couloir à la cloison translucide pour pénétrer dans la chambre de Goya âgé. Saura invente ainsi une alternative au montage dont il convient de mesurer la portée. Car si le montage est une construction du temps, ainsi que Deleuze l'a montré, son absence manifeste constitue une négation de l'accomplissement du temps. La continuité qui s'instaure entre différentes nappes du passé rend impossible la construction d'un temps chronologique.

Si le film défie les lois de la chronologie, c'est qu'il suit le cheminement tortueux d'une mémoire humaine qui oblitère ou modifie les faits passés, confond les époques, circule librement, par association d'idées, entre les différentes régions du passé. L'anachronie devient le signe d'une expérience subjective du temps. Saura renoue ici avec la modernité d'écriture qui caractérise tout un pan de son œuvre. On pense à des films comme Peppermint frappé (1967), la Prima Angélica (1973), Cría cuervos (1975), Elisa vida mía (1977), dans lesquels le cinéaste, marqué par la découverte de Buñuel, s'ingénie à brouiller la perception du spectateur par des effets de dédoublement des personnages, de rupture du continuum spatio-temporel, par la remise en cause permanente des liens de causalité entre les événements narrés, par la confusion constante des mondes réels, des mondes rêvés ou des mondes fantasmés. Des œuvres qui, en rupture avec le réalisme de ses premiers films, explorent l'intériorité des personnages et résistent aux interprétations faciles.

\section{A-chronie}

Dans Goya en Burdeos, l'anachronie débouche assez logiquement sur une sorte d'a-chronie, c'est-à-dire un effacement de l'Histoire dont nous avons déjà eu l'occasion d'écrire qu'il était caractéristique du film. C'est un effacement qui reste partiel car l'ancrage contextuel demeure, assuré par la présence de personnages historiques proches de Goya, tel Moratín ou Godoy, dont les portraits sont rapidement esquissés, on l'a vu, ou à l'occasion de quelques rares séquences qui semblent renouer ponctuellement avec la tradition naturaliste du film en costume. L'une d'elles met en scène la petite communauté des libéraux en exil à Bordeaux, réunie dans le café chocolaterie du compatriote aragonais de Goya, Braulio Poc. Une autre évoque une réception chez les ducs d'Osuna. Ce sont les quelques concessions du film au plaisir du dépaysement et à la quête d'exotisme qui ont fait de tout temps l'attrait du cinéma historique. Dans ces rares instants, l'art du décorateur et du costumier s'exprime magnifiquement. Trop, peut-être, au goût de Saura qui laisse percer de l'ironie voire un certain agacement dans le commentaire qu'il fait de sa collaboration avec le décorateur Pierre Louis Thévenet : "Thévenet qui était quelqu'un de merveilleux, mais qui au début n'a rien compris au film, il n'a rien compris parce qu'il voyait des choses très concrètes. Il avait commencé par faire de magnifiques dessins d'intérieurs, d'extérieurs. J'étais fasciné... Mais, je lui ai dit : «Ce n'est pas cela, je veux faire précisément le contraire de tout cela ${ }^{14} \ldots$..

Le contraire, ce sont ces décors minimalistes qui le plus souvent tiennent de l'abstraction. C'est à peine s'ils portent la marque d'une époque. Du reste, peu d'accessoires entourent Goya. La mise en relief du personnage tient aussi à ce dépouillement. Le cinéaste se serait 
alors inspiré du peintre qui procédait de la sorte : ses fonds ressemblaient à des décors de théâtre, peu léchés en apparence, comme si l'effort de l'artiste tendu vers la représentation des personnages avait délaissé l'accessoire, c'est-à-dire la réalité palpable d'une époque et d'un lieu. Sans doute faut-il entendre les propos que Saura fait tenir à Goya tandis qu'il contemple les Ménines de Velázquez comme la définition de l'un des principaux enjeux du film :

Voilà la peinture que je voulais faire. Une peinture qui paraisse inachevée, légère, qui semble avoir été faite sans effort. Hors de tout temps, de tout espace, de tout lieu [...] au-delà de toute réalité palpable et physique. Cette autre réalité, celle qui naît de la peinture, miroir déformant de la vie, reflet d'un instant, réalité magique où tout est possible ${ }^{15}$.

Ainsi le Goya de Saura semble-t-il évoluer hors des contraintes du temps et de l'espace, dans le monde tantôt hallucinatoire, tantôt onirique né de son esprit tourmenté.

\section{L'anachronisme comme un mode de pensée de I'histoire de l'art}

21 La séquence aperturale, véritable plongée dans le monde intérieur du peintre est particulièrement révélatrice de cet entrelacs des temps ${ }^{16}$. Le film s'ouvre sur un cauchemar de Goya. En un long travelling, la caméra balaye un sol rougeâtre et livre au regard du spectateur les restes d'un sacrifice: une tête de bœuf, des coutelas ensanglantés, un amas de cordes, des récipients remplis de sang. Puis s'élevant, elle découvre la dépouille d'un animal traîné sur le sol par des mains invisibles. La carcasse de l'animal est bientôt hissée sur une structure de bois en un simulacre de crucifixion. L'image convoque certainement le souvenir de quelques-unes des œuvres les plus brutales de Goya, en particulier certaines gravures des Désastres de la guerre où s'offre le spectacle macabre de corps en lambeaux réduits à des monceaux de chair. Toutefois, c'est la vision d'une œuvre n'appartenant pas à Goya qui s'impose peu à peu. Car s'il est un tableau auquel l'image de la carcasse de bœuf crucifiée fait penser irrésistiblement c'est bien au célèbre Bœuf écorché de Rembrandt. On ne saurait s'étonner de cet hommage à un peintre à l'égard duquel Goya n'a cessé de dire sa dette. Mais, pourquoi avoir choisi cette toile si particulière? Peut-être parce que peint en 1655, Le boeuf écorché est l'une de ces œuvres à partir desquelles s'écrit l'Histoire de l'art. Dans son ouvrage sur Rembrandt, Christopher Wright écrit «Le bœuf écorché peint en 1655 est devenu une icône de l'hagiographie rembranesque. Ce tableau a eu une importance particulière pour les peintres du XXème siècle et il a joué un rôle éminent dans le développement des artistes russes émigrés tel Soutine ${ }^{17}$. » Le tableau de Rembrandt n'aura cessé d'être copié, repris, relu, pastiché. De Delacroix à Bonvin, de Daumier à Soutine, d'Antonio Saura à Bacon, innombrables sont les peintres qui ont puisé leur inspiration auprès de cette œuvre exceptionnelle. C'est cette sorte de communauté d'artistes, de «famille spirituelle $»^{18}$, réunie par-delà les époques, par une même fascination pour ce tableau, que convoquent les premières images du film. Quand la caméra s'approche au plus près de la carcasse et que les entrailles se transforment par un effet de morphing pour donner naissance au visage de Goya, on songe plus particulièrement au tableau célèbre de Francis Bacon, Figure with meat (1954), une toile montrant le pape Innocent $X$ trônant entre deux pièces de bœuf, qui s'inspire du chef-d'œuvre viscéral de Rembrandt et d'un portrait du pape Innocent X par Velázquez. Douloureuse métaphore de la solitude devant la mort, le 
tableau vivant ainsi composé par Saura exprime parfaitement le sentiment que le cinéaste prête à Goya tandis qu'il vit à Bordeaux ses derniers jours. Il n'en demeure pas moins que l'anachronisme a de quoi surprendre. Le maître de Fuendetodos est habité par une vision qui des années plus tard harcèlerait le peintre britannique. Saura fait bien plus que suggérer une filiation entre Goya et Bacon que beaucoup d'historiens d'art jugent probable. Il nous invite à une analyse rétrospective de l'œuvre du maître aragonais à la lumière de Bacon. Acceptons donc l'invitation que nous fait Saura et, avec lui, empruntons en rebours le long cheminement de l'Histoire de l'art. L'incipit de Goya en Burdeos n'accomplirait-il pas à sa façon le parcours insolite qui conduirait de l'abstraction à la figuration? Jacques Terrasa fait sienne cette hypothèse lorsqu'il décrit les premières secondes du film:

Goya en Burdeos commence par des images abstraites: durant 40 secondes, des taches de lumière, dispersées de manière aléatoire sur la toile rectangulaire, offrent une composition informelle, évoquant la texture de quelque tableau matiérique comme ceux que peignait Antoni Tàpies dans les années 1950. C'était l'époque où le jeune Carlos Saura délaissait la photographie pour se consacrer au cinéma, l'époque où son frère Antonio - dont le nom apparaîtra au générique quelques secondes plus tard : «A mi hermano Antonio »- devenait l'un des plus éminents représentants de cette nouvelle peinture abstraite. Noires et blanches au départ, les premières images du film vont bientôt se teinter de rouge, rappelant la palette d'un Millares, un Canogar ou un Feito, pour citer quelques artistes informalistes moins connus que les deux précédents ${ }^{19}$.

Saura aurait ainsi radicalisé dès les premières secondes de son film le propos de Malraux qu'il reproduit à sa conclusion: "Après Goya, commence la peinture moderne ». Mais, sans doute s'agit-il moins d'inverser le cours du temps que d'inscrire, dès ces premiers plans, l'œuvre de Goya dans le flux ininterrompu de l'Histoire de la peinture. Goya puise son art à la source des maîtres que sont Rembrandt et Velazquez - ce que l'on savait déjà - et annonce en même temps la modernité de Bacon et d'Antonio Saura. L'hommage à Goya débute donc par un hommage à la capacité qu'ont les grands créateurs d'instaurer un dialogue fécond avec l'Histoire de l'art. C'est cette pratique artistique, faite d'emprunts, de réminiscences et d'intuitions, riches d'un dialogue constant aussi bien avec les œuvres du passé qu'avec celles qui sont à venir, qu'entend exalter Saura. Certes, le cinéaste s'empare ici d'une idée qu'un certain nombre d'historiens de l'art ont développée au cours du vingtième siècle. Henri Focillon a montré que dans les grandes œuvres d'art, il y a toujours des temporalités différentes qui se superposent et s'entrelacent. Dans le dernier chapitre de La Vie des formes, intitulé «Les formes dans le temps", il exprime avec force sa conviction de ce que les événements historiques ne s'ordonnent pas selon une évolution linéaire : «L'Histoire est généralement un conflit de précocité, d'actualités et de retards. L'histoire de l'art nous montre, juxtaposées dans le même moment, des formes lentes, retardataires, contemporaines de formes hardies et rapides ${ }^{20}$.» Le présent de la création mélange à la fois le passé et le futur. Ce que l'historien d'art Daniel Arasse résume en affirmant qu'une œuvre d'art est un objet anachronique : «Donc dans le temps même de sa production, une œuvre d'art peut très bien mélanger les temps et faire elle-même de l'anachronisme. L'idée d'une pureté du temps linéaire dans l'Histoire de l'art n'a pas de sens $»^{21}$. À cet égard, l'incipit de Goya à Bordeaux est une clé de lecture pour l'ensemble du film. Il est riche de cette conviction selon laquelle la pensée créatrice doit s'affranchir des découpages artificiels de l'Histoire, car ainsi que l'écrit encore Daniel Arasse, « la peinture est un objet historique produit à 
un certain moment dans des conditions précises, mais la pensée de la peinture peut aller au-delà des conditions historiques de la pensée de son temps ${ }^{22}$.»

Evoquer, comme nous le faisions dans le titre de cette communication, "le regard anachronique » porté par le film de Carlos Saura sur Goya et son époque, c'est reconnaître d'emblée la profonde originalité de cette œuvre au regard de la pratique courante du cinéma d'Histoire. Au terme de cette réflexion, Goya en Burdeos nous apparaît comme une œuvre hybride à mi-chemin entre le genre de l'essai et celui de la biographie historique. Saura tout à la fois adopte la démarche du réalisateur de films historiques qui puise dans la documentation des éléments susceptibles d'assurer un effet d'époque et s'en démarque en s'émancipant de la logique positive souvent adoptée par l'historien, voire en procédant à un effacement partiel ou total du contexte historique. La vie de l'artiste, les liens qu'il a tissés avec ses contemporains, le regard qu'il a porté sur son époque, l'ensemble des anecdotes qui ont jalonné son existence, tout ce qui en définitive l'inscrit dans un temps révolu, le cantonne à un passé irrémédiablement disparu, tout cela s'estompe. Seules comptent les traces laissées par le peintre, son œuvre qui, bien que conçue il y a deux siècles, demeure en quelque sorte notre contemporaine en ceci que nous l'admirons et qu'elle nous parle encore, qu'elle ne cesse de nous interpeller. Goya en Burdeos fait la démonstration que lorsque le personnage historique (c'est-à-dire en fait le personnage inscrit dans l'histoire, le personnage historicisé) disparaît, subsiste encore l'essentiel : son œuvre.

\section{NOTES}

1. Goya en Burdeos, réalisation: Carlos Saura, scénario: Carlos Saura, photographie: Vittorio Storaro, musique: Roque Baños, direction artistique: Pierre Louis Thévenet, décors: Luis Ramírez, Montage : Julio Juaniz, producteur : Andrés Vicente Gómez, principaux interprètes : Francisco Rabal, José Coronado, Dafné Fernández, Eulalia Ramón, Maribel Verdú.

2. Dictionnaire historique de la langue française, sous la direction de Alain Rey, Paris, Dictionnaires Le Robert, 1992.

3. Dictionnaire culturel en langue française, sous la direction de Alain Rey, Paris, Dictionnaires Le Robert, 2005.

4. Michel de Certeau, L'Ecriture de l'Histoire, Paris, Gallimard, 1975.

5. Paul Ricoeur, «Histoire et rhétorique ", Diogène, $n^{\circ}$ 168, 1994, p. 25. Cité par Roger Chartier, "La vérité entre fiction et histoire», in Antoine de Baecque, Christian Delage, De l'histoire au cinéma, Paris, Editions complexes, 1998, p. 42.

6. Michel Cieutat, «Les biopics de 1930 à 1960, ou le dopage d'une nation », Positif, n540, février 2006, p. 82

7. «El concepto general es hacer una película sobre Goya alejada del naturalismo. »

8. Carlos Saura, «Goya (Goya en Burdeos), Apuntes para una película sobre Goya escrita y dirigida por Carlos Saura », Artigrama, Universidad de Zaragoza, 1994. p. 12.

9. «Las libertades que me he tomado en mi Goya son consecuencia de mi personal visión del personaje. No quise hacer ni una película histórica, ni un compendio (un résumé) de su obra, ni mucho menos un análisis psicológico del personaje -aunque de todo ello haya en mi película-, 
sino entresacar (extraire) de su amplia vida aquellas imágenes que consideraba claves » Goya ante Saura », in Carlos Saura, « Goya ante Saura », www.el-mundo.es/magazine/m7/textos/goya1.html, consultation : 10-02-2007.

10. Nancy Berthier, « Carlos Saura ou l'art d'hériter », in Jean-Pierre Castellani (coord.), Goya en Burdeos de Carlos Saura, Paris, Editions du Temps, 2005, pp. 191-239.

11. «Que si han tenido una relación, que si la han tenido, que si no... Pero ¿Qué más da? [...] la película es una película imaginativa [...] » Carlos Saura, « Entrevista », in Jean-Paul Aubert, JeanClaude Seguin (dir.), De Goya à Saura. Echos et résonances, Le Grimh, Lyon, 2005, p. 249

12. Jacques Fauqué, Ramón Vallanueva Etcheverria, Goya y Burdeos 1824-1828, Oroel, Zaragoza, 1982.

13. Yannick Mouren, Le Flash-back, Armand Colin Cinéma, Paris, 2005, p. 28.

14. "Thévenet que era una persona maravillosa, pero que no entendió nada de la película al principio, que no entendió nada porque él veía cosas muy concretas, empezando por unos dibujos preciosos de interiores, de exteriores... Estaba fascinado... Pero le dije : «Mira, es que no es por ahí, quiero hacer todo lo contrario de esto.... » Carlos Saura, «Entrevista », in Jean-Paul Aubert, Jean-Claude Seguin (dir.), De Goya à Saura. Echos et résonances, Le Grimh, Lyon, 2005, p. 254.

15. «Esa era la pintura que yo quería hacer. Una pintura que pareciera incacabada, ligera, con la apariencia de hacerse sin esfuerzo. Fuera de todo tiempo, espacio y lugar. [...] más allá de toda realidad palpable y física. Esta otra realidad, la que nace de la pintura, espejo deformante de la vida, reflejo de un instante, realidad mágica donde todo es posible. »

16. Nous ne ferons qu'évoquer succinctement dans ces lignes une séquence que nous avons déjà eu l'occasion de commenter de manière plus détaillée : Jean-Paul Aubert, « Goya en Burdeos : un "portrait imaginaire », in Jean-Paul Aubert, Jean-Claude Seguin (dir.), De Goya à Saura. Echos et résonances, Le Grimh, Lyon, 2005, pp. 163- 190. On lira également avec beaucoup de profit ce qu'écrit au sujet de cette même séquence Jacques Terrasa : «Les Territoires de la chair. Incipit de Goya en Burdeos ", in Elisabeth Delrue, Goya. Image de son temps. De l'Espagne des Lumières à l'Espagne libérale, Indigo, publication de Université de Picardie, 2005.

17. Christopher Wright, Rembrandt, Citadelle \& Mazenod, Paris, 2000, p. 278.

18. L'expression appartient à l'historien d'art Henri Focillon, in La Vie des formes, Paris, Leroux, 1934, p. 79.

19. Jacques Terrasa, « Les Territoires de la chair. Incipit de Goya en Burdeos », article cité, p. 122

20. Henri focillon, La Vie des formes, p. 83

21. Daniel Arasse, Histoires de la peinture, Gallimard, Paris, 2006 [Denoël, 2004], p. 227.

22. Ibid., p. 243.

\section{RÉSUMÉS}

Cet article a pour objet l'anachronisme dans le film historique. Il s'appuie sur l'étude du long métrage Goya à Bordeaux, réalisé en 1999 par le cinéaste espagnol Carlos Saura. Cette biographie filmée du peintre Francisco de Goya fait mieux que composer avec l'anachronisme inhérent à toute reconstitution historique. Il l'assume et le revendique. La mise en cause de la chronologie et l'effacement de l'Histoire prennent sens dans la perspective d'une réflexion originale sur l'Histoire de l'art conçue comme un dialogue entre les œuvres et entre les époques. 
Este artículo está dedicado al problema del anacronismo en las películas históricas. Se basa en el estudio de Goya en Burdeos, un largo metraje rodado en 1999 por el director de cine español Carlos Saura. Esta biografía de Goya no sólo se acomoda con el anacronismo propio de la reconstitución histórica sino que lo asume y lo reivindica. El cuestionamiento de la cronología y el desinterés por la Historia cobran un nuevo sentido en el marco de una reflexión original sobre la Historia del arte concebida como un diálogo entre las obras y entre las épocas.

INDEX

Index chronologique : XXe siècle

Index géographique : Espagne

Mots-clés : cinéma, Histoire, anachronisme, Francisco de Goya, Carlos Saura

\section{AUTEUR}

JEAN-PAUL AUBERT

Université de Nice-Sophia Antipolis, CIRCPLES, EA 3159 